\title{
SYSTEMIC CANNABIDIOL DOES NOT REDUCE COMPOUND 48/80-INDUCED ITCHING BEHAVIOR IN MICE
}

\author{
Hatice Demirel ${ }^{1}$, Elif Baksın¹, Ece Önay Özgür ${ }^{1}$, Ruhan Deniz Topuz², Ahmet Ulugöl ${ }^{2}$
}

${ }^{1}$ Trakya University School of Medicine, Edirne, TURKEY

${ }^{2}$ Department of Medical Pharmacology, Trakya University School of Medicine, Edirne, TURKEY

\section{ABSTRACT}

Aims: Cannabinoids are chemical compounds including natural cannabinoids found in the Cannabis plant, their synthetic counterparts, and endocannabinoids. Cannabidiol, a phytocannabinoid derived from the Cannabis plant, exerts anticonvulsant, anxiolytic, anti-inflammatory, neuroprotective, analgesic effects. Although there are many similarities between the pathophysiological mechanisms of pain and itch, researches that investigate the effect of cannabinoids on itching are insufficient. Here, we aimed to examine the antipruritic effect of cannabidiol and the contribution of spinal cannabinoid receptors.

Methods: Male Balb/c mice, weighing 20-30 g, were used. Itching behavior was produced by intradermal injection of compound 48/80 (100 $\mu \mathrm{g} / 50 \mu \mathrm{l})$; cannabidiol $(1,3,10 \mathrm{mg} / \mathrm{kg}$, ip) was administered 30 minutes before compound 48/80 injections. Then, scratching of the injected site by the hind paws was videotaped for 30 minutes. Locomotor performances were assessed using a rotarod apparatus.

Results: Cannabidiol had no effect on compound 48/80-induced itching behavior at any dose given; moreover, cannabidiol did not produce any impairment on motor function. AM-251, a cannabinoid receptor type 1 antagonist, and AM-630, a cannabinoid receptor type 2 antagonist were administered intrathecally to observe the contribution of spinal cannabinoid receptors to the antipruritic action of cannabidiol. We observed that cannabidol did not possess any effect on itching behaviour.

Conclusion: Our results indicate that systemic administration of cannabidiol does not attenuate compound 48/80 induced itching behavior in mice.

Keywords: Cannabidiol, compound 48-80, pruritus

\section{INTRODUCTION}

Pruritus can be described as an unpleasant and strange sensation of irritation, which may also involve tingling, biting or burning that initiates itching in the skin in related areas. Many similarities between the neuronal pathways and the pathophysiological mechanisms of pain and itching have been proposed; itching sensation is transmitted to spinal cord's dorsal horn by primary afferent $C$ fibers, and then to the brain by spinothalamic pathways $(1,2)$. Glutamate is suggested as the principal excitatory neurotransmitter in the spinal cord not only for pain but also for itching; similarly, descending inhibition is involved in the development of both pain and itching sensations $(3,4)$. Similar symptoms to allodynia, hyperalgesia and abnormal pain also occur in pathologi- cal itching conditions $(2,5)$. Taken together, the spinal cord seems to be an attractive target for developing new drugs against pruritus (6-8).

Cannabinoids are chemical compounds including natural cannabinoids found in the Cannabis plant (phytocannabinoid), their synthetic counterparts, and substances that make up the endogenous cannabinoid system (endocannabinoid) synthesized in the body (9). Cannabinoid receptor type $1(\mathrm{CB} 1)$ and cannabinoid receptor type 2 (CB2) receptors are the primary targets of all cannabinoids. Cannabis has been used for medical purposes, especially to treat pain, until the Marihuana Tax Act of 1937 which decreased its use rapidly. In recent years, there have been changes in policies which led to an increase in the use of medical cannabis in many countries $(10,11)$. 
In addition to pain states, cannabinoids have been proposed as potential antipruritic drugs. Cannabinoid receptor agonists have been shown to reduce histamine-induced scratches, whereas cannabinoid receptor antagonists provoked pruritic responses via CB1 receptors $(12,13)$. Attenuation of itching replies by augmenting endocannabinoid tonus via the inhibition of the endocannabinoid degradative enzymes, such as fatty acid amide hydrolase and monoacylglycerol lipase which is a different promising method for treating pruritus (1416). Our research group also indicated that the synthetic cannabinoid agonist WIN 55,212-2 exerts dose-dependent antipruritic effects and this effect is partially mediated by spinal cannabinoid receptors CB1 $(17,18)$.

Unlike most of the other cannabinoids, the non-psychoactive phytocannabinoid cannabidiol exhibits little or no orthosteric binding potential at cannabinoid CB1 and CB2 receptors (19). However, cannabidiol has extensive therapeutic properties, including anticonvulsant, anxiolytic, anti-inflammatory, neuroprotective, analgesic effects, etc (20). Thus, the purpose of this study is to investigate the antipruritic effect of cannabidiol in compound 48/80-induced itching behavior in mice and whether spinal cannabinoid receptors are involved in this action.

\section{MATERIAL AND METHODS}

The investigations were approved by the institutional ethics committee of Trakya University. This experiment was carried out in young male Balb/c mice (obtained from Center of the Laboratory Animals, Trakya University), weighing 20-30 g ( $\mathrm{n}=8$ for each group). Animals were maintained under a 12-12 h light/dark cycle at a constant temperature of $21 \pm 2{ }^{\circ} \mathrm{C}$ with food and water ad libitum. Mice were housed in a group of 8 per cage and the experiments were conducted in a quiet room between 10:00 and 17:00. Animals were allowed to acclimate to laboratory conditions for one week before the experiments were performed; each mouse was tested only once. All procedures involving mice were carried out in strict accordance with "Guide for the Care and Use of Laboratory Animals" published by National Academy of Sciences (21).

Itching behavior was produced by intradermal injection of compound 48/80 (100 $\mu \mathrm{g} / 50 \mu \mathrm{l})$; compound $48 / 80$ is a well-known histamine releasing agent which produce scratches subsequent to mast cell degranulation. Scratching injected site by the hind paws was ac- cepted as the itching behavior; mice scratched several times after compound 48/80 injection, and this reaction is counted as one bout of scratching. Scratches were video recorded in a quiet room, and then counted for $30 \mathrm{~min}$. Testing was accomplished according to previously described procedures (22-24).

Locomotor performances were assessed using a rotarod apparatus (Commat, Ankara, Turkey). The animals were acclimatized to the apparatus before the assessments. Then, mice were placed on the drum rotating at $16 \mathrm{rpm}$ and the performance time until the mice fell from the drum. 180 seconds cut-off frequency was adjusted before the experiments.

Groups of eight mice each received increasing doses of cannabidiol (1, 3, $10 \mathrm{mg} / \mathrm{kg}$, ip). Cannabidiol was administered 30 min before compound 48/80 injections. Then, the cannabinoid CB1 receptor antagonist AM-251 (1g/mouse) and the cannabinoid CB2 receptor antagonist AM-630 (4g/mouse) were given intrathecally 10 min prior to cannabidiol administration in order to determine whether spinal cannabinoid receptors are involved in the effect of cannabidiol on itching behavior.

Cannabidiol was purchased from Tocris (UK), while compound 48/80, AM-251 and AM-630 from Sigma-Aldrich (St Louis, MO, USA. Compound 48/80 was dissolved in physiological saline, whereas cannabidiol, AM-251, and AM-630 were given in 20\% dimethyl sulfoxide (DMSO), 1\% Tween $80,1 \%$ ethanol, and $78 \%$ saline. Cannabidiol was administered intraperitoneally in a volume of $0.05 \mathrm{ml} / 10 \mathrm{~g}$ body weight of mice, AM-251 and AM-630 were injected intrathecally (51/ mouse), and compound 48/80 was given intradermally $(100 \mu \mathrm{g} / 50 \mu \mathrm{l})$. Previous studies guided doses and treatment (23-25).

Differences in the number of scratches in durations on the rotating rod were evaluated using analysis of variance and were followed by Bonferroni's multiple comparison tests. All data are expressed as mean SEM; $\mathrm{p}<0.05$ was considered to be significant for all experiments. 


\section{RESULTS}

Treatment with cannabidiol (1, 3, $10 \mathrm{mg} / \mathrm{kg}$, ip) had no effect on compound 48/80-induced itching behavior at any dose given (Figure 1). $3 \mathrm{mg} / \mathrm{kg}$ dose of cannabidiol seemed to reduce the number of scratches, but this reduction was not statistically significant ( $\mathrm{p}=0.4499)$.

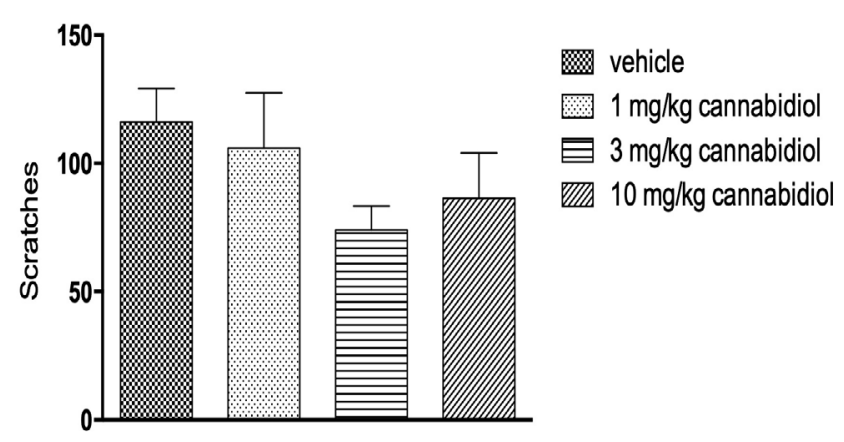

Figure 1: The effect of cannabidiol $(1,3,10 \mathrm{mg} / \mathrm{kg}$, ip) on the number of scratches.

The effect of cannabidiol $(1,3,10 \mathrm{mg} / \mathrm{kg}$, ip) on locomotion was evaluated in the rotarod test, where no significant change on motor function was observed (Figure 2).

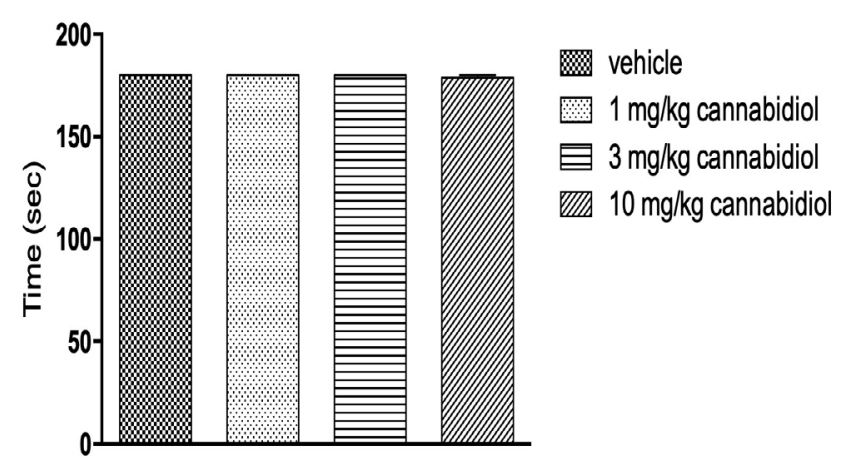

Figure 2: The effect of cannabidiol (1, 3, $10 \mathrm{mg} / \mathrm{kg}$, ip) on locomotion.

AM-251, a CB1 antagonist; AM-630, a CB2 antagonist, were going to be administered intrathecally to observe the contribution of spinal cannabinoid receptors to the antipruritic action of cannabidiol, but they were not given when we observed cannabidiol did not exert any effect on itching behavior.

\section{DISCUSSION}

The non-psychoactive phytocannabinoid cannabidiol is a compound that does not produce typical subjective effects of marijuana. Different from the classical cannabinoids, including those found in the Cannabis plant, cannabidiol exhibits very low affinity with CB1 and CB2 (19). On the other hand, possible therapeutic uses of cannabidiol include analgesia, epilepsy, anxiety, schizophrenia, depression, and many more. Other than activity on cannabinoid receptors, there are multiple potential mechanisms underlying this wide spectrum of potential beneficial effects of cannabidiol. Firstly, recent findings also indicate that cannabidiol is a negative allosteric modulator of CB1 (26). Moreover, cannabidiol has been shown to be a transient receptor potential cation channel subfamily $\mathrm{V}$ member 1 (TRPV1) agonist which desensitize TRPV1 even at lower concentrations (27). Additionally, its pharmacological effects have been assigned to peroxisome proliferator-activated receptor (PPAR) agonism, intracellular calcium release and serotonin $1 \mathrm{~A}$ receptor (5-HT1A) agonism (28, 29). Cannabidiol also appears to act via fatty acid amide hydrolase (FAAH) inhibition and augment endocannabinoid levels (28).

Considering similarities between pain and itching sensations and the potent analgesic effect of cannabidiol in different types of pain states, one would expect cannabidiol to show antipruritic action in mice (1-5). Its ineffectiveness in compound 48/80-induced itching behavior may be attributed to above-mentioned mechanisms unrelated to classical cannabinoid actions $(26,27,29)$. Moreover, variations in physiological state, age, strain, and sex of the mouse, dose range and volume, route of administration and method of restraint are among the factors influencing this kind of behavioral research. Differences in assessment methods and existing animal models also seem to be important; for example, applying the pruritogens intradermally into the rostral part of the neck has been indicated not to discriminate pain and itching sensations but may give false positive results with analgesic drugs (23). Furthermore, the characteristics of the environment and the history of the subjects, such as exposure to stress, are suggested to interfere with the activity of cannabinoids in behavioral studies (30). Since cannabinoids have been proposed to excite circadian clock neurons and the activity of the endocannabi- 
noid system is profoundly modulated by circadian rhythmicity, the ineffectiveness of cannabidiol in reducing scratches may have also resulted from the timing of drug administration $(32,33)$.

As we mentioned before, spinal cord appears promising for developing novel antipruritic drugs (6-8). In addition to the well-known involvement of spinal opioid receptor, gastrin-releasing peptide receptor and N-methyl-D-aspartate glutamate receptor in pruritus, serotonin, histamine, substance $\mathrm{P}$ and bradykinin receptors are among potential itching treatment targets $(6,7)$. It has also been demonstrated that blockade of spinal cannabinoid CB1 receptors partially reverse the antipruritic effect of synthetic cannabinoid WIN 55,212-2 (17). Here, we weren't able to investigate the contribution of spinal cannabinoid receptors since systemic cannabidiol had no effect on itching behavior.

Cannabidiol is used in some countries against pruritus; however, our findings suggest that systemic administration of cannabidiol does not diminish compound 48/80-induced itching behavior in mice. Recently, reduced pruritus and improved pain scores have been reported in patients with epidermolysis bullosa after combined treatment with tetrahydrocannabinol and cannabidiol (33). The effectiveness of cannabidiol in alleviating itching behavior should be investigated with further studies by using different itching models and experimental protocols.

Ethics Committee Approval: This study was approved by the Scientific Researches Committee of Trakya University School of Medicine.

\section{Informed Consent: N/A}

Conflict of Interest: The authors declared no conflict of interest.

Author contributions: Concept: RDT, AU. Design: HD, EB, EÖÖ, RDT, AU. Supervision: RDT, AU. Resources: RDT, AU. Materials: HD, EB, EÖÖ, RDT, AU. Data collection and/or processing: HD, EB, EÖÖ, RDT, AU. Analysis and/or Interpretation: HD, EB, EÖÖ, RDT, AU. Literature Search: HD, EB, EÖÖ, RDT, AU. Writing Manuscript: RDT, AU. Critical Review: HD, EB, EÖÖ, RDT, AU.

Financial disclosure: This work was supported by a grant from Trakya University Research Council (TÜBAP-2017/44).
Acknowledgements: We thank K. Duvan Aydemir for her technical support for performing behavioral tests.

\section{REFERENCES}

1. Davidson S, Giesler GJ. The multiple pathways for itch and their interactions with pain. Trends in Neurosciences 2010;33:550-8.

2. Ikoma A, Cevikbas F, Kempkes C et al. Anatomy and neurophysiology of pruritus. Semin Cutan Med Surg 2011;30:64-70.

3. Koga K, Chen T, Li X-Y et al. Glutamate acts as a neurotransmitter for gastrin-releasing peptide-sensitive and insensitive itch-related synaptic transmission in the mammalian spinal cord. Mol Pain 2011;7:47.

4. Gotoh Y, Omori Y, Andoh T et al. Tonic inhibition of allergic itch signaling by the descending noradrenergic system in mice. J Pharmacol Sci 2011;115:417-20.

5. Ross SE, Mardinly AR, McCord AE et al. Loss of inhibitory interneurons in the dorsal spinal cord and elevated itch in Bhlhb5 mutant mice. Neuron 2010;65:88698.

6. Cevikbas F, Steinhoff M, Ikoma A. Role of spinal neurotransmitter receptors in itch: new insights into therapies and drug development. Cns Neurosci Ther 2011;17:742-9.

7. Kuraishi Y. Potential new therapeutic targets for pathological pruritus. Biol Pharm Bull 2013;36:1228-34.

8. Ross SE. Pain and itch: insights into the neural circuits of aversive somatosensation in health and disease. Current Opinion in Neurobiology 2011;21:880-7.

9. Ulugol A. The endocannabinoid system as a potential therapeutic target for pain modulation. Balkan Med J 2014;31:115-20.

10. Grotenhermen F, Muller-Vahl K. The therapeutic potential of cannabis and cannabinoids. Dtsch Arztebl Int 2012;109:495-501.

11. Schrot RJ, Hubbard JR. Cannabinoids: medical implications. Annals of Medicine 2016;48:128-41. 
12. Dvorak M, Watkinson A, McGlone F et al. Histamine-induced responses are attenuated by a cannabinoid receptor agonist in human skin. Inflammation Research 2003;52:238-45.

13. Schlosburg JE, O’Neal ST, Conrad DH et al. CB1 receptors mediate rimonabant-induced pruritic responses in mice: investigation of locus of action. Psychopharmacology 2011;216:323-31.

14. Schlosburg JE, Boger DL, Cravatt BF et al. Endocannabinoid modulation of scratching response in an acute allergenic model: a new prospective neural therapeutic target for pruritus. Journal of Pharmacology and Experimental Therapeutics 2009;329:314-23.

15. Spradley JM, Davoodi A, Gee LB et al. Differences in peripheral endocannabinoid modulation of scratching behavior in facial vs. spinally-innervated skin. Neuropharmacology 2012;63:743-9.

16. Tosun NC, Gunduz O, Ulugol A. Attenuation of serotonin-induced itch responses by inhibition of endocannabinoid degradative enzymes, fatty acid amide hydrolase, and monoacylglycerol lipase. Journal of Neural Transmission 2015;122:363-7.

17. Bilir KA, Anli G, Ozkan E et al. Involvement of spinal cannabinoid receptors in the antipruritic effects of WIN 55,212-2, a cannabinoid receptor agonist. Clin Exp Dermatol 2018;43:553-8.

18. Todurga ZG, Gunduz O, Karadag $\mathrm{CH}$ et al. Descending serotonergic and noradrenergic systems do not regulate the antipruritic effects of cannabinoids. Acta neuropsychiatrica 2016;28:321-6.

19. Pertwee RG. The diverse CB1 and CB2 receptor pharmacology of three plant cannabinoids: Delta(9)tetrahydrocannabinol, cannabidiol and Delta(9)-tetrahydrocannabivarin. Brit J Pharmacol 2008;153:199215.

20. Iffland K, Grotenhermen F. An update on safety and side effects of cannabidiol: a review of clinical data and relevant animal studies. Cannabis Cannabinoid Res 2017;2:139-54.
21. National Research Council (US) Committee for the Update of the Guide for the Care and Use of Laboratory Animals. Guide for the Care and Use of Laboratory Animals. 2011 (cited 2018 Sep 5). Available from: URL: https://www.ncbi.nlm.nih.gov/books/NBK54050/ doi: 10.17226/12910.

22. Gunduz O, Topuz RD, Todurga ZG et al. Effect of activation of the GLT-1 transporter by a beta-lactam antibiotic on serotonin-induced scratching behavior in mice. Neurophysiology 2015;47:36-9.

23. Ilkaya F, Yesilyurt O, Seyrek M et al. The false-positive responses of analgesic drugs to the intradermal serotonin- and compound 48/80-induced scratches as an animal model of itch. Acta Neurobiol Exp 2016;76:23443.

24. Saglam G, Gunduz O, Ulugol A. Blockade of cannabinoid CB1 and CB2 receptors does not prevent the antipruritic effect of systemic paracetamol. Acta Neurologica Belgica 2014;114:307-9.

25. Aydemir KD, Gunduz O, Ulugol A. Effects of a nociceptin receptor antagonist on experimentally induced scratching behavior in mice. Neurophysiology 2017;49:130-4.

26. Laprairie RB, Bagher AM, Kelly MEM et al. Cannabidiol is a negative allosteric modulator of the cannabinoid CB1 receptor. Brit J Pharmacol 2015;172:4790805.

27. Bisogno T, Hanus L, De Petrocellis L et al. Molecular targets for cannabidiol and its synthetic analogs: effect on vanilloid VR1 receptors and on the cellular uptake and enzymatic hydrolysis of anandamide. Brit J Pharmacol 2001;134:845-52.

28. Campos AC, Moreira FA, Gomes FV et al. Multiple mechanisms involved in the large-spectrum therapeutic potential of cannabidiol in psychiatric disorders. Philos T R Soc B 2012;367:3364-78.

29. Russo EB, Burnett A, Hall B et al. Agonistic properties of cannabidiol at 5-HT1a receptors. Neurochemical Research 2005;30:1037-43.

30. Viveros MP, Marco EM, File SE. Endocannabinoid system and stress and anxiety responses. Pharmacology Biochemistry and Behavior 2005;81:331-42. 
31. Acuna-Goycolea C, Obrietan K, Van Den Pol AN. Cannabinoids excite circadian clock neurons. Journal of Neuroscience 2010;30:10061-6.

32. Hanlon EC, Tasali E, Leproult R et al. Circadian rhythm of circulating levels of the endocannabinoid 2- arachidonoyl glycerol. J Clin Endocr Metab 2015;100:220-6.

33. Schrader NHB, Duipmans JC, Molenbuur B et al. Combined tetrahydrocannabinol and cannabidiol to treat pain in epidermolysis bullosa: a report of three cases. Br J Dermatol 2018 Oct 22. [Epub ahead of print] 